\title{
INCREASING THE ACCURACY OF TIME-TABLED CONDITION MONITORING
}

\author{
Binling Jin and Suzanne M. Embury \\ Department of Computer Science, University of Manchester, \\ Oxford Road, Manchester, M13 9PL, UK \\ $\{$ Bjin, SEmbury\}@cs.man.ac.uk
}

\begin{abstract}
Many applications benefit from the ability to monitor the value of conditions over large, possibly distributed data sets. However, mechanisms to achieve this typically impose a performance overhead that the owners and users of such systems may be unwilling to accept. A compromise solution is to allow system owners to decide at what times they will allow computational resources to be given over to condition monitoring and at what times they will not. This allows the system owner to maintain maximum throughput rates during the most important periods of the day or week, while still reaping the benefits of a limited form of condition monitoring. The monitoring is "limited" because the results will contain inaccuracies. However, in some applications, even inaccurate information about conditions will be of value, if it can be provided at minimal cost to the organisation in question. This approach is called non-intrusive condition monitoring.

In this paper, we discuss a variety of methods for reducing the inaccuracy of nonintrusive condition monitoring in distributed information systems. The methods employ a range of temporal reasoning techniques, and promise differing degrees of improvement in accuracy. They also each impose different costs on the operation of the system as a whole. We present some experimental results which support our categorisation of the costs and benefits of the proposed methods.
\end{abstract}

Keywords: Integrity Constraint Checking, Condition Monitoring, Distributed Database Systems

\section{INTRODUCTION}

The ability to monitor changes in conditions of interest as they occur in information systems is useful in a variety of ways. Sometimes, such a facility can be a required part of a specific application, as in the case of the Active_INEKS project, which used condition monitoring techniques to inform epidemiologists of the appearance of potential cancer clusters in a changing medical data set [2]. Two other examples of such applications are robot manipulators [13] and stock market control [6]. However, condition monitoring can also be useful to the administra-

The original version of this chapter was revised: The copyright line was incorrect. This has been corrected. The Erratum to this chapter is available at DOI: 10.1007/978-0-387-35693-8_16 
tors of information systems, in integrity maintenance and usage monitoring for tuning and security purposes, for example.

Unfortunately, however, generalised condition monitoring facilities (such as those provided by an active rule mechanism $[3,12]$ ) can involve significant performance penalties, and this has resulted in a reluctance to use such features in real applications [10]. Part of the problem is the difficulty of creating a truly efficient implementation of a very general condition monitoring mechanism ${ }^{1}$. But another aspect of the problem (and one that is much less often acknowledged) is the time at which the processing involved in monitoring the condition takes place - typically, just after an event of interest has occurred. These "events of interest" typically occur during times when system activity is at its highest - that is, at times when the systems are involved in significant amounts of processing. However, in mission-critical systems and systems which carry out revenue-generating work, these times are exactly the worst times for performing this additional processing. The throughput that can be achieved by these systems is often a key factor in the competitive success of the organisation which owns them. Taking processing time away for more "peripheral" applications can impose a cost that is unacceptable to the owning organisation.

The problem, therefore, is not so much the fact that condition monitoring systems impose a performance overhead; instead, it is a matter of when that performance overhead is imposed. In many systems, the administrators are very happy to allow such additional processing to take place overnight, when the system is quiet, even if no spare processing capacity is available on the system during normal business hours (for example). When system owners are unwilling to pay the price of fully accurate condition monitoring, therefore, they might instead be open to the use of some more limited facility that operates only during certain specified periods of time. The price of this, of course, is some loss in accuracy: some changes in the condition's value will not be detected, while others will be incorrectly timestamped. Whether the benefits are worth the cost will depend on the application in question.

In previous work, we have explored the possibilities for providing a condition monitoring system for use in distributed information systems (DISs), that allows the owners of the component systems to specify when they are willing to give up processing resources to the monitoring system [9]. Our long-term goal is to use this facility to track the occurrence of data defects in DISs, in order to support information quality improvement programmes [14], but our basic system (which uses an approach based on polling) can be applied wherever a generalised distributed condition monitoring facility is required. We use the term non-intrusive

\footnotetext{
${ }^{1}$ For example, Kotz-Dittrichand Simon report the perceptions of developers, who have compared the performance of versions of applications implemented using triggers with versions implemented without, and who have found the versions using triggers to be "significantly slower" [10].
} 
condition monitoring to refer to this style of condition monitoring, since it attempts to avoid intruding into periods of important business processing.

One of the main challenges inherent in the provision of non-intrusive condition monitoring is to try to make the best use of the system time that is available, so that the changes in the conditions are recorded as accurately as possible. In part, this requires careful choice of the execution plan that is used to partition the work of evaluating the condition amongst the various components of the DIS [9]. Some execution plans will make better use of the available time, and therefore will result in more accurate records of the condition's value than others. However, assuming that the distribution of the work between the sites is fixed (i.e. that an execution plan has been chosen), the accuracy of the result can still be affected by the manner in which that plan is executed.

For instance, techniques used to increase the accuracy of distributed query processing (DQP) can also be employed in our setting (since non-intrusive condition monitoring is essentially a form of DQP). Gupta and Widom, for example, have described several means of improving the accuracy of distributed query processing by exploiting information stored (or cached) locally [4], while others have exploited parallel query evaluation techniques for this purpose [1,5]. However, as we shall show in this paper, it is also possible to reduce inaccuracy by making use of temporal reasoning techniques. To the best of our knowledge, such techniques have not been applied to this problem before.

In this paper, we present four different ways to increase the accuracy of nonintrusive condition monitoring, each using temporal reasoning techniques at varying levels of sophistication and each presenting a different cost-benefit trade-off in terms of the improvement in accuracy gained. We begin by presenting in more detail the causes of the inaccuracy in this context (Section 2), before describing the methods we propose to use to counter it (Section 3). We then outline the experimental framework that we have used to explore the cost-benefit trade-offs implied by each method (Section 4 ) and draw conclusions as to which of the methods are most likely to be of value in practice (Section 5).

\section{CAUSES OF INACCURACY IN CONDITION MONITORING}

Before we can begin to try to improve the accuracy of our approach to condition monitoring, it is necessary to understand exactly what it is that causes the inaccuracy. In fact, there are two different types of inaccuracy that must be dealt with. One is the incompleteness of the results, which is inherent in any approach based on polling. The other is the inaccuracy caused by the need to avoid accessing certain systems during certain periods of time, and the delays in query processing that this inevitably introduces. We will now briefly discuss these two sources of inaccuracy. 


\subsection{Incompleteness Inherent in Approaches based on Polling}

Typical applications of condition monitoring are in diagnosis, treatment planning and monitoring [7] and strategic planning [8]. In this type of application, it is necessary to record the changing state of a condition over time, rather than take specific action when its value changes from false to true. For example, in order to reason about the root causes of poor data quality, and to plan and monitor the progress of any improvement programme, it is necessary to be able to analyse when certain undesirable data patterns have occured in the database, and for how long they were present [9]. However, when conditions are monitored by polling, we record only the state of the condition at certain discrete points in time, rather than producing a continuous record of its state over a period. This discontinuity of results makes analysis of the changing patterns of conditions in the data difficult. For example, Figure 1 shows the results obtained by polling a condition $C$ over a period. The state of the condition was polled at times $t_{1}, t_{3}, t_{4}, t_{6}$ and $t_{7}$, with the condition proving to be true at times $t_{3}, t_{4}$ and $t_{6}$ and false at other times. But, if the user asks about the value of $C$ at times $t_{2}$ or $t_{5}$, what are we to answer? Similarly, how can we calculate the total number of data defects present in DB during one month? We will discuss several ways to deal with these problems in Section 3.1.

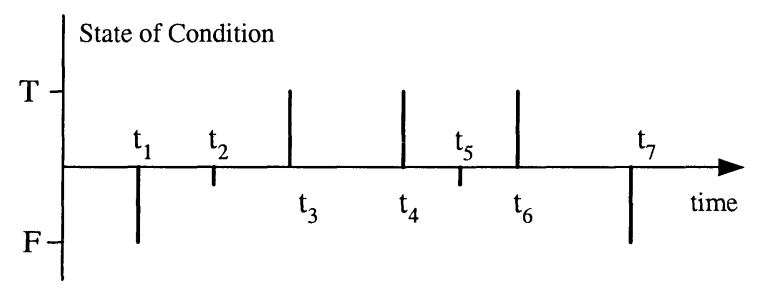

Figure 1. Example Results from Condition Monitoring by Polling

\subsection{Inaccuracies due to Time Delays}

The second cause of inaccuracy in non-intrusive condition monitoring is delay. In any form of distributed query evaluation, the computation of the query result may be delayed because of the need to ship partial data sets between sites, and because some data resources may be temporarily unavailable. In non-intrusive condition monitoring, there is an additional source of delay: systems may refuse to respond to query requests at times when their owners do not wish them to devote resources to condition monitoring. For all these reasons, there will usually be a gap between the time at which the condition changes its value (which we call the change time, or $C T$ for short) and the time at which the condition monitor detects this change (the detected time or DT). 
For example, suppose we are monitoring a condition $C$ that involves order handling data stored at two sites $\left(s_{1}, s_{2}\right)$, and we wish to store the changing state of the condition at site $s_{3}$. The condition to be monitored is that there should be no orders on which some product is given a different price to that recorded in the product catalogue. Suppose further that information about orders is stored at site $s_{1}$, while the product catalogue table is stored at site $s_{2}$. One possible way of evaluating $C$ is given by the following sequence of operations:

1. $O p_{1}$ : ship the orders table from $s_{1}$ to $s_{2}$

2. $O p_{2}$ : evaluate the condition query at $s_{2}$

3. $O p_{3}$ : ship the result from $s_{2}$ to $s_{3}$

Suppose that the execution times for $O p_{1}, O p_{2}$ and $O p_{3}$ are $d_{1}, d_{2}$ and $d_{3}$ respectively. Suppose further that a tuple $t p$ is inserted into the database at time $t_{1}$ that causes $C$ to become true, and that the system next begins to poll the value of $C$ at time $t_{2}$. If there are no other delays, and all sites involved are continuously available, then the process of condition evaluation would occur as illustrated in Figure 2, and the change in the value of the condition would finally be recorded at time $t_{2}+d_{1}+d_{2}+d_{3}$. This is inaccurate, since the condition actually became true much earlier than the recorded time. It may also be inaccurate in another sense, since the condition might have become false once more by the time its "value" is recorded at $s_{3}$. This inaccuracy is usually greater than this, due to the additional delays needed to ship data between sites and to wait for sites to become available to execute operations. Several methods for reducing this form of inaccuracy are discussed in Section 3.2.

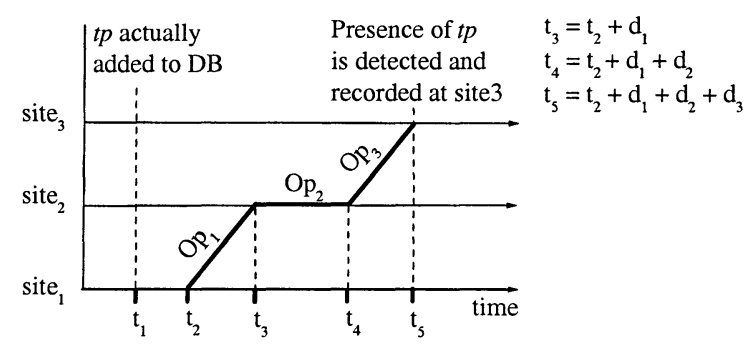

Figure 2. Time Delays in Distributed Condition Monitoring 


\section{INCREASING THE ACCURACY OF POLLED RESULTS}

Having outlined the causes of the inaccuracies, we will now discuss how to address them. We first present some methods for interpreting a sequence of polled results, to give the impression that a continuous data set has been recorded, before discussing four methods of reducing inaccuracy due to processing delays.

\subsection{Interpreting Polled Results}

In order to generate an apparently complete description of the state of a polled condition over time, it is necessary to "guess" at what value the condition might have held in between the polling times. For example, one very simple approach to making such a "guess" is to assume that if a condition is found to have the same value in two consecutive polled results, then its value has not changed at any time in between. Furthermore, if a condition's value is found to have changed between consecutive polls, then we might assume that the change occurred immediately prior to the latter of the two polls. Under these assumptions, the polled results shown in Figure 1 would be interpreted as the continuous condition record shown in Figure 3. This approach has the advantage of simplicity but, as it is not very likely that the condition always does change its value immediately before it is polled, this method will often produce inaccurate results.

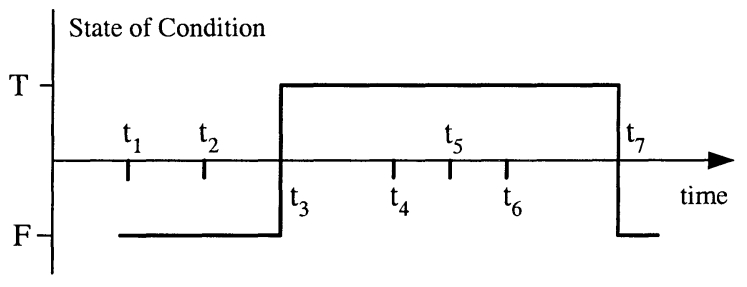

Figure 3. Interpreting Approach 1

An alternative technique is to assume that the condition alters its value exactly half way between consecutive polled results which indicate a change. The effects of applying this assumption to the interpretation of the polled results from Figure 1 are shown in Figure 4.

Yet another approach is to interpret the polled results not as a Boolean function, but as a function that gives, for any point in time, the probability that the condition was true or false at that time. At points in time where a polled result has been computed and the condition has been found to be true, the function would return a probability of $100 \%$. Similarly, when the condition is known to be false, the function would return a result of $0 \%$. In between polled results, the probability 


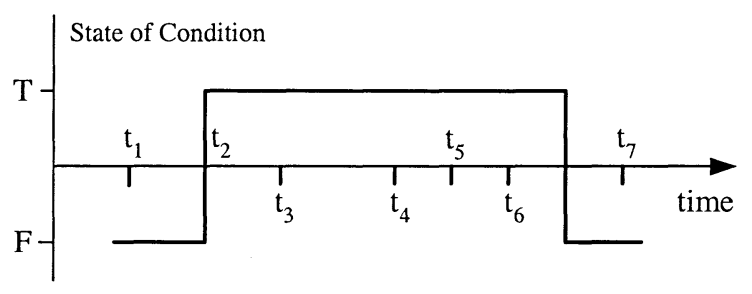

Figure 4. Interpreting Approach 2

decreases or increases with distance from the time of the polling. Under this approach, the example polled results would be interpreted as shown in Figure 5.

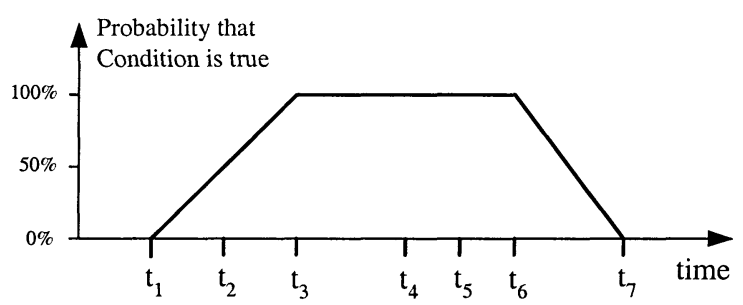

Figure 5. Interpreting Approach 3

\subsection{Reducing Inaccuracy Caused by Delays}

In Section 2.2, we saw how delays can occur during distributed condition monitoring due to the need to transfer data between sites or the temporary inaccessibility of sites. These delays mean that query results may not be an accurate reflection of the state of the condition by the time they are received and recorded by the destination site. In this section, we present four different ways to reduce the inaccuracy caused by these delays.

\subsubsection{Method One: Timestamp Query Results Locally.}

For certain condition queries (strictly speaking, for certain execution plans of certain queries), the final result is actually known some time before it is received and timestamped by the condition monitoring site. For instance, suppose a polled result is computed by one site $s_{x}$ at time $t_{1}$ and is then shipped to another site to be recorded. If it takes $d$ seconds to ship the result, then it will be timestamped with a detected time of $t_{1}+d$. In this situation, we would obtain a more accurate timestamp for the results if we allowed $s_{x}$ to add its own local timestamp to the data before shipping. This timestamp could then be used by the recording site, as the timestamp for the polled condition's value, even though it was actually received rather later. 
For example, Figure 6 shows a typical sequence of events when a distributed condition is monitored. Suppose that the value of the condition $(C)$ changes at time $t_{1}$, and that the condition is next evaluated by some local site at time $t_{2}$. It then takes until time $t_{3}$ for the results to be shipped to the condition monitoring site. By shipping the time at which the result was generated $\left(t_{2}\right)$ along with it, we can timestamp the results with a detection time that is much closer to the change time $\left(t_{1}\right)$ than would otherwise be the case.

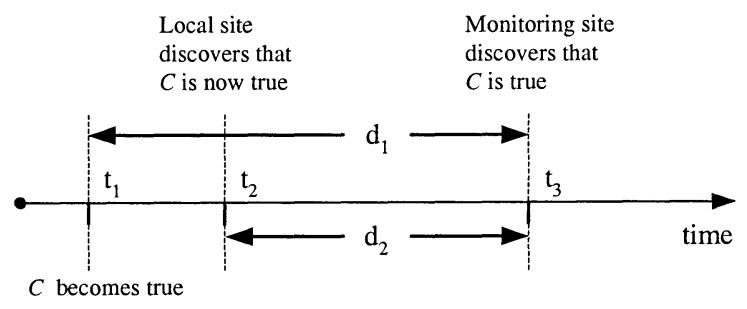

Figure 6. Timestamp Polled Results Locally

The advantage of this approach is that it does not impose much of an overhead on query processing, since it only needs to timestamp the polled result as they are computed at local sites. However, this method only solves part of the problem. There are other sources of inaccuracy which can be eradicated, as we shall now discuss.

\subsubsection{Method Two: Timestamp Based on the Source Relations.}

The method discussed above is predicated on the fact that the value of the condition may be known to some local site $s_{x}$ of the distributed system before it is known to the condition monitoring site. However, since there may be a delay between the change time of the condition and the time at which the change in the state of the condition is detected at $s_{x}$, this approach will still produce an inaccurate timestamp in many cases. We will now discuss how we can improve on this, beginning with a description of a simplified form of the approach for queries consisting of a single relational algebra operator, and then generalising this to relational algebra queries of arbitrary complexity. First, however, we introduce some notation. For any relational algebra operator $q$, the result of $q$ is called its target relation $(T R(q))$, and the relations over which the query is executed are called its source relations $(S R s(q))$. The time at which a relation $r$ was last updated is denoted $L U T(r)$, while the most recent update time of a set of relations $R s$ is denoted MaxLUT $(R s)$.

Suppose that the condition monitoring system begins to evaluate a query $q$ (consisting of the application of a single relational algebra operator) at time $t$. We know that $T R(q)$ will have been unchanged since $\operatorname{MaxLUT}(S R s(q))$. Therefore, the timestamp for the polled result can be given as $\operatorname{Max} L U T(S R s(q))$. This basic 
idea can be applied to each individual operator within more complex queries, so that the timestamps on the intermediate relations are used as the "time of last update" to calculate the timestamps of the operators which make use of them. The timestamp for the full condition is that which is computed for the target relation of the query as a whole.

This method can make the final timestamp on monitored conditions more accurate, but this benefit comes at a cost. First, it requires some means of detecting and recording the time at which each source relation was last updated. In some cases, the data management software in use at a site may already keep track of this information, but in others additional processing overhead must be expended in collecting it. Second, the process of evaluating the condition query is now complicated by the need to compute the timestamps. This may also impose a slight additional performance penalty on condition evaluation.

\subsubsection{Methods Three and Four: Timestamp Individual Tuples.}

The two methods described above attempt to bring the timestamp given to a polled result closer to the time at which the change in the condition's value actually occurred. However the timestamps so obtained are still not completely accurate, since not all changes to source relations will actually result in a change to the condition's value. In addition, any change in the truth value of a monitored condition must be due to some insertion, deletion, or update of some specific tuple, so the change time is closely related to the times at which these updates occur. Therefore, in order to improve the accuracy still further, we could make use of timestamps on individual tuples, rather than on tables as a whole. We will now describe two ways to timestamp the individual tuples and to calculate the time at which the condition's truth value changes.

Timestamp Insertions and Updates: One way to represent timestamps on individual tuples is to extend the relations involved in any monitored conditions with an extra attribute (start_time) in which to store the time at which each tuple was last updated or inserted into the relation. This implies that the intermediate and target relations of the condition query must also include a start_time attribute. During condition evaluation, the start_time of the result relation can be calculated using the standard approach for performing temporal operations on time-varying relations [11]. Although it is more usual to use time intervals to represent the valid time of individual tuples, such intervals can be simulated by assuming that the end_time of each valid time interval is null.

For example, consider two relations, $R_{1}$ and $R_{2}$, with schemas $\left(X_{1}, \ldots, X_{n}\right.$, start_time $)$ and $\left(Y_{1}, \ldots, Y_{m}\right.$, start_time $), n, m \geq 1$, respectively. Attributes $X_{1}, \ldots, X_{n}$ and $Y_{1}, \ldots, Y_{m}$ are time-varying attributes. If $R_{3}=R_{1} \bowtie R_{2}$ then $R_{3}$ can be calculated using the following algorithm: 
For each $<x_{1}(i), \ldots, x_{n}(i), T S_{i}>\in R_{1}$ and $<y_{1}(j), \ldots, y_{m}(j), T S_{j}>\in R_{2}$,

Then

$<x_{1}(i), \ldots, x_{n}(i), y_{1}(j), \ldots, y_{m}(j), T S_{i j}>\in R_{3}$, where $T S_{i j}=\operatorname{MAX}\left(T S_{i}, T S_{j}\right)$, and $M A X\left(T S_{i}, T S_{j}\right)$ returns the most recent timestamp of $T S_{i}$ and $T S_{j}$.

One major advantage of this method is that it can accurately calculate the change time of a condition that are the result of the insertion of new tuples. However, if the change is caused by the update of an existing tuple, then change time computed by this method may be later than the actual change time. The reason for this is that the condition's change time is calculated according to the last updated time of tuples (recorded as the start_time), whereas the actual change may have resulted from some earlier update.

Moreover, this method cannot accurately calculate the time of changes caused by deletions, since it does not store the information about when tuples are deleted. Another disadvantage is that query evaluation will be slower, since we must also calculate the new start_time attributes. In addition, extra disk space may be required in order to store the start times for the base and intermediate relations. However, some DBMSs (e.g. ORACLE8) will automatically timestamp tuples when they are updated or inserted into the DB. Thus, this method may not require any additional disk space in practice.

Timestamp Deletions: In order to calculate a condition's change time completely accurately, it is also necessary to store information about when tuples are deleted. For each of the base relations involved in monitored conditions, we now require an additional relation to store details of when tuples are deleted. This relation is referred to as the transaction log of deletions (TLD). If the schema of a base relation $R$ is $\left(X_{1}, \ldots, X_{n}\right.$, start_time $)$ and only the attributes $X_{1}, \ldots, X_{m}$ (where $m \leq n$ ) are involved in monitored conditions, then the schema of the $T L D$ for $R$ is: $\left(X_{1}, \ldots, X_{m}\right.$, start_time, end_time). Here, the start_time and end_time attributes store the times at which each tuple is inserted into the DB and deleted from the $\mathrm{DB}$, respectively.

If a base relation $R$ has a $T L D$ called $S$, then when a tuple $\left\langle x_{1}, \ldots, x_{n}, t_{s}\right\rangle$ is deleted from $R$ at time $t_{e}$, a tuple $\left\langle x_{1}, \ldots, x_{m}, t_{s}, t_{e}\right\rangle$ will be inserted into $S$. Insertion of a tuple leaves the end_time null, while update of of a tuple is considered to be equivalent to deletion followed by an insertion. From the two relations $R$ and $S$, we can now generate a complete description of the history of $R$ in terms of valid time intervals using the query $\left(\Pi_{X_{1}, \ldots, X_{m}, t_{s}, n u l l \rightarrow t_{e}}(R)\right) \cup S$ We refer to this generated relation as the complete transaction log $(C T L)$.

By this method, when the condition monitoring system polls a condition, it first will generate the $C T L s$ for each base relation involved in the condition query and then evaluate the query over these generated relations, rather than the base relations. This produces the final condition result, complete with valid time 
intervals. In order to do this, it is necessary to perform temporal operations over the time intervals stored in the $C T L s$ while performing the standard relational operations over the time-varying attributes. As discussed previously, this can be achieved using well-known methods for temporal query evaluation [11].

However, this method imposes a very severe cost, both in terms of the additional data required to record tuple's update information and the additional processing time that is required for query evaluation. A further cost is that condition evaluation using this approach will repeatedly calculate information about past condition changes, which have already been recorded by the condition monitoring system. These negative effects can be ameliorated if we periodically delete tuples from the $T L D s$. But, when should this deletion be carried out? And which tuples can safely be deleted? A variety of answers might be given to these questions, and we suggest only one here. If the condition monitoring system begins to evaluate a set of condition queries at time $t_{x}$, and has fully evaluated each of them by time $t_{y}$, then all transaction log tuples which refer only to the queries in this set and which have an end time that is earlier than $t_{x}$ may be deleted.

The advantage of this approach to condition evaluation is that it accurately identifies the true change time of each condition. However, as we have mentioned, a significant amount of additional disk space may be required to store all the transaction $\log$ relations. Moreover, in order to maintain the transaction logs, we must monitor any updates to the base relations so that the transaction log relations can be kept up to date. This can cause a significant slow-down in the system's ability to process revenue-generating transactions and perform other essential business processing, which may be unacceptable to the system's owners and users.

A further disadvantage of full timestamping of tuples is that the transaction logs are likely to contain a significant amount of redundant data. For example, they will record details of all updates to tuples even when the attributes involved in the monitored condition have not been changed. The disk space required to store this redundant data may be prohibitively high, especially if updates to attributes that are not involved in any monitored condition occur very frequently. The obvious solution to this problem is to timestamp changes at an even greater level of granularity than individual tuples, i.e. at the level of individual attributes of tuples. However, the costs of this form of timestamping will clearly be even higher than those of timestamping at the level of whole tuples, and it is far from clear that the benefits obtained will be worthwhile. We do not consider this approach any further in this paper.

\section{EVALUATION}

We have presented four methods to improve the accuracy of condition monitoring by polling. Compared to the naive approach of timestamping the results as they arrive at the monitoring site, we would expect each of these four methods to 
increase the accuracy of condition monitoring, but to differing degrees. We would further expect that they would each impose differing costs in terms of their effect on normal business processing and their disk space requirements. We need to understand the cost-benefit trade-off implied by each method, so that the most appropriate one can be chosen in any given situation. We have therefore undertaken an experimental study of these methods, in an attempt to quantify and compare their costs and benefits.

The expected benefit of each of the methods is an increase in the accuracy of condition monitoring. In order to quantify this benefit, we define the inaccuracy of a method applied over a period of time to be equivalent to the amount of time during that period when the condition monitoring system has recorded a false positive or a false negative result for some condition. A false positive result occurs when the condition monitoring systems states that, the condition was true when in fact it was false. Similarly, a false negative result occurs when the condition monitoring system states that the condition was false when it was actually true. By totalling up the length of time during which an inaccurate result was recorded, we can gain some indication of the inaccuracy inherent in the condition monitoring method used.

The expected costs are twofold: a possible decrease in the rate at which normal business transactions can be processed (due to the need to perform housekeeping operations for condition monitoring) and the amount of additional disk space that is necessary to record information required for condition monitoring alone. We quantify these two costs by measuring the number of non-condition-monitoring transactions that can be executed over the period being studied (called the TransactionNumber), and the amount of disk space the database as a whole occupies at the end of the period being studied (called the SizeOfDB). Based on the form of each method, we would expect the methods to incrementally improve the accuracy of condition checking (i.e. Method 4 is better than Method 3, which is better than Method 2, which is in turn better than Method 1), but that the costs will also incrementally increase in the same manner.

In order to test this prediction, and to measure these costs and benefits in a (semi-) realistic application, we have developed a simple order handling system, distributed over three sites, and a condition monitoring facility based on our work on non-intrusive condition monitoring [9]. The distributed database holds information on orders, products, customers, employees and managers, and totals $62 \mathrm{Mb}$ in size. We have also developed an experimental framework which allows us to execute transactions against the database and record the actual state of the condition, while monitoring the condition using one of our methods to record the detected state of the condition for comparison. Each run of the system lasts for a period of 12 hours, and the rate at which violations of conditions are created and removed can be controlled as a parameter of the method. 
We have used this framework to compare the Inaccuracy, TransactionNumber and SizeOfDB parameters for each of the four methods discussed in this paper, when monitoring the condition relating to order prices mentioned earlier. In order to provide a baseline for our comparisons, we have also measured the costs and benefits of the simple "naive" approach to condition monitoring, in which results are timestamped as they arrive at the condition monitoring site (referred to as Method 0).

The results of our experiments are illustrated in Figure 7. As this figure shows, our initial predictions regarding the relative benefits and costs of the methods are borne out by the experimental results. The most sophisticated of the methods (Method4) does indeed produce a dramatic reduction in the inaccuracy of condition monitoring, when compared with the naive method. However, it also imposes a heavy cost, both in terms of the additional disk space required (approximately 50\% of the actual size of the database) and in terms of the dramatic cut in the number of other transactions that can be executed.

Based on these results, Method Two would appear to be the best compromise, giving a good increase in accuracy, but without imposing any significant costs in terms of disk space or transaction processing rates. However, in practice, the choice of method to apply would depend on the competing desires of the users of the system for high transaction rates and for high accuracy in condition monitoring.

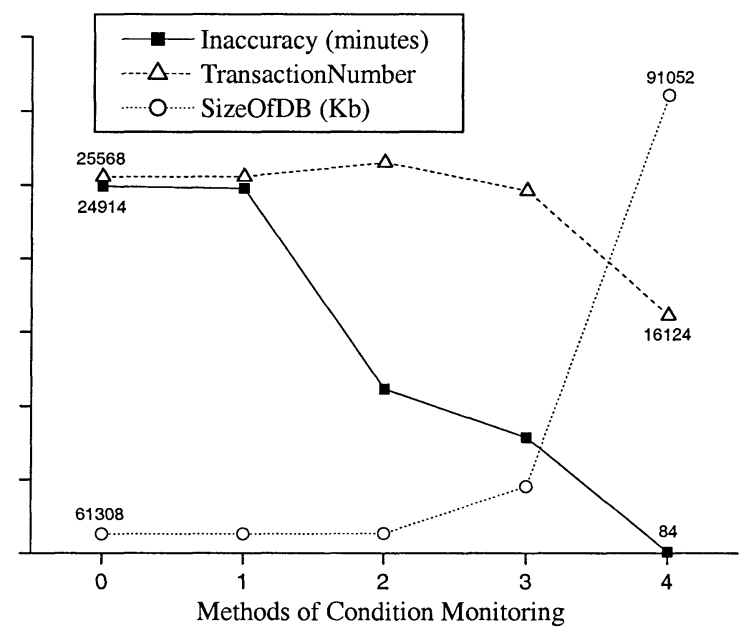

Figure 7. Benefits Versus Cost 


\section{CONCLUSIONS}

We have discussed some of the problems inherent in a non-intrusive approach to condition monitoring based on polling, the most significant of which is its inaccuracy. While other approaches to condition monitoring (most notably those based on active rules and triggers) may provide accurate results, they impose a performance penalty that the users of revenue-generating systems may be unwilling to accept. This is especially the case when the condition monitoring facility is required to record the status of a condition over time, for the purposes of tactical and strategic planning, rather than to react quickly as particular situations arise. For such systems, normal business processing is the key priority, and any information that can be garnered by the condition monitor must be obtained in such a way that this processing is not slowed down to any great degree. Where this is the case, there may be no option but to adopt polling as a means of condition monitoring, inaccuracies and all.

In this paper, we have presented several methods for reducing the inaccuracy of condition monitoring by polling, and have compared their relative strengths and weaknesses in terms of the improvement in accuracy they provide, and their effects on business processing and disk space. Our experimental results indicate that significant improvements in accuracy can be achieved by the application of temporal reasoning techniques at the individual tuple level. But, this improvement comes with a price: effectively, it imposes many of the same costs as a full active rule approach to condition monitoring. However, our experimental results suggest that significant improvements in accuracy can be achieved by means of simple table-level temporal reasoning, with little overall cost to the system as a whole.

Our longer term aim is to use the non-intrusive condition monitoring approach (with the table-level temporal reasoning) to monitor data quality levels in distributed information systems. The system will detect data defects and will provide a detailed record of the times at which they appear within and disappear from the system, with the minimal disruption to crucial revenue-generating data processing. By analysing the polled results, we hope to find the root causes of the poor data quality, so that system managers can better plan and monitor the progress of long term data quality improvement programmes.

\section{REFERENCES}

[1] P. Apers, C. D. Berg, J. Flokstra, P. Grefen, M. Kersten, and A. Wilschut. PRISMA/DB: A Parallel, Main Memory Relational DBMS. IEEE Transactions on Knowledge and Data Engineering, 4(6):541-554, December 1992.

[2] H.-J. Appelrath, H. Behrends, H. Jasper, and V. Kamp. Active Database Technology Supports Cancer Cluster Clustering. In W. Litwin and T. Risch, editors, Applications of Databases, First International Conference, ADB-94, Vadstena, Sweden, June 21-23, 1994, Proceedings. Lecture Notes in Computer Science, Vol.819, pages 351-364. Springer, 1994. 
[3] S. Ceri and J. Widom. Deriving Production Rules for Constraint Maintainance. In D. McLeod, R. Sacks-Davis, and H.-J. Schek, editors, 16th International Conference on Very Large Data Bases, August 13-16, 1990, Brisbane, Queensland, Australia, Proceedings, pages 566-577. Morgan Kaufmann, 1990.

[4] A. Gupta and J. Widom. Local Verification of Global Integrity Constraints in Distributed Databases. In P. Buneman and S. Jajodia, editors, Proceedings of the 1993 ACM SIGMOD International Conference on Management of Data, Washington, D.C., May 26-28, 1993, pages 49-58. ACM Press, 1993.

[5] U. Herzog and R. Schaarschmidt. Parallel Execution of Integrity Constraint Checks. In CIKM '95, Proceedings of the 1995 International Conference on Information and Knowledge Management, November 28 - December 2, 1995, Baltimore, Maryland, USA, pages 82-89. ACM, 1995.

[6] B. Hollifield, R. Miller, P. Sandås, and J. Slive. Adverse Selection and Competitive Market Making: Empirical Evidence from a Limit Order Market. The Review of Financial Studies, 14:705-734, 2001.

[7] J. Hunter and N. McIntosh. Knowledge-Based Event Detection in Complex Time Series Data. In W. Horn, Y. Shahar, G. Lindberg, S. Andreassen, and J. Wyatt, editors, Artificial Intelligence in Medicine, Joint European Conference on Artificial Intelligence in Medicine and Medical Decision Making, AIMDM'99, Aalborg, Denmark, June 1999, Proceedings. Lecture Notes in Computer Science, Vol. 1620, pages 271-280. Springer, 1999.

[8] R. Hutton. The Impact of Information Technology on Condition Monitoring. In B. Rao, editor, The 5th International Conference on Profitable Condition Monitoring: Fluids and Machinery Performance Monitoring, Harrogate, UK, December 3-4, 1996, pages 23-35. Mechanical Engineering Publications Ltd, 1996.

[9] B. Jin and S. Embury. Non-Intrusive Assessment of Organisational Data Quality. In E. Pierce and R. Katz-Haas, editors, The 6th International Conference on Information Quality (IQ2002), Cambridge, MA, USA, November 2001, pages 398-411, 2001.

[10] A. Kotz-Dittrich and E. Simon. Active Database Systems: Expectations, Commercial Experience, and Beyond. In N. W. Paton, editor, Active Rules in Database Systems, pages 367-404. Springer, 1999.

[11] S. B. Navathe and R. Ahmed. Temporal Relational Model and a Query Language. Information Sciences, 49(1-3):147-175, 1989.

[12] N. W. Paton and O. Diaz. Active Databases. ACM Computing Surveys, 31(1):63-103, March 1999.

[13] B. Rao. Handbook of Condition Monitoring. Elsevier Advanced Technology, Oxford,UK, 1996.

[14] T. Redman. Data Quality for the Information Age. Artech House, Boston, USA, 1996. 